\title{
CARTOGRAPHIE DES HABITATS NATURELS DES ESTRANS ET ZONES HUMIDES LITTORALES
}

\author{
Aurélie Dehouck ${ }^{1}$, Virginie Lafon ${ }^{1}$, Nicolas Baghdadi ${ }^{2}$, Guillaume Clos $^{1,3}$, Danielle Ducrot ${ }^{3}$, Samantha Yeo ${ }^{4}$, Marie-Lise \\ Benot $^{4}$, Cécile Curti ${ }^{1}$, Pierre-Guy Sauriau ${ }^{5}$, Stéphane Kervella ${ }^{1}$ \\ 1: GEO-Transfert, EPOC, Université de Bordeaux, aurelie.dehouck@u-bordeaux.fr \\ 2: IRSTEA, TETIS, Montpellier \\ 3: CESBIO, Toulouse \\ 4: BIOGECO, Université de Bordeaux \\ 5: LIENSs, Université de La Rochelle
}

\begin{abstract}
Résumé
A l'interface entre terre et mer, les zones humides littorales sont des environnements dont le fonctionnement écosystémique est contraint par les forçages climatiques et anthropiques. Par le biais des directives européennes et des plans de gestion, ces milieux sont suivis et cartographiés à échéance régulière dans le but d'asseoir leur état écologique. Différentes stratégies de cartographie basées sur l'exploitation d'images satellite sont adoptées selon les objectifs à atteindre : (1) cartographie exhaustive des faciès/habitats intertidaux (type Natura 2000), (2) cartographie de l'herbier à Zostera noltii (Directive Cadre sur l'Eau), (3) cartographie des habitats des prés-salés, (4) suivi de l'évolution naturelle des paysages et de la végétation en lien avec des événements accidentels ou volontaires (actions de dépoldérisation).
\end{abstract}

Cet article dresse un panorama des stratégies de cartographie exploitant des données optiques et radar à Haute (SPOT5) et Très Haute Résolution Spatiale (Pléiades, TerraSAR-X) mises au point pour répondre aux besoins d'observation et d'expertise pour la gestion intégrée des milieux littoraux lagunaires.

Mots-clés : habitats, littoral, estran, prés-salés, images satellites

\begin{abstract}
In between land and ocean, tidal flats and coastal wetlands functioning and evolution are constrained by climatic and anthropic-driven forces. Monitoring of these coastal areas takes place in the frame of European environmental directives and integrated management plans aiming at addressing their ecological status. Several satellite image-based mapping strategies can be implemented to answer these various needs : (1) exhaustive habitat mapping over intertidal areas (Natura 2000 oriented), (2) mapping of seagrass meadows of Zostera noltii (Water Framework Directive), (3) mapping of salt marsh habitats, (4) monitoring of landscape and vegetation changes driven by accidental or voluntary depolderisation events.
\end{abstract}

This paper addresses developed mapping strategies based on both optical and radar High Resolution (SPOT-5) and Very High resolution (Pléiades, TerraSAR-X) images that were defined for the monitoring and management of coastal areas.

Keywords: habitats, coastal areas, tidal flats, salt marshes, satellite imagery

\section{Introduction}

Cet article résume les travaux récents portant sur la cartographie de la biodiversité des écosystèmes littoraux de la façade atlantique à partir de données satellitaires à Haute Résolution (HR) et Très Haute Résolution (THR). Les milieux d'intérêt sont les lagunes tidales soumises à l'action de la marée qui incluent les zones intertidales (estran) et supratidales (marais littoraux ou prés salés). La gestion des zones humides littorales s'inscrit dans un contexte réglementaire européen (Directive Cadre sur l'Eau, Directive Habitat i.e. Natura 2000) et local par le biais des plans de gestion spécifiques à chaque site protégé, réserve naturelle ou parc marin. Au travers des travaux présentés dans cet article, nous verrons comment les données THR (Pléiades notamment) sont intégrées dans des stratégies de cartographie résolument orientées utilisateur dans le but de répondre au mieux aux spécifications des gestionnaires du littoral.

\section{Données et méthodes}

\subsection{Cartographie de l'herbier à Zostères (Zostera noltii)}

La cartographie des herbiers constitue à l'heure actuelle un important enjeu de gestion qui explique en partie l'intérêt de la communauté internationale pour le développement de méthodes dédiées, notamment à partir des technologies spatiales (Bargain et al., 2013, Finn, 2008). Dans le but de développer une approche opérationnelle, reconnue pour la cartographie de l'état de l'herbier au sens de la Directive Cadre sur l'Eau (DCE), une classification hiérarchique fondée sur une image optique multispectrale estivale est retenue. Cette approche assimile directement des observations de 
terrain (points et contours GPS, photos numériques), recueillies pendant la saison estivale (Figure 1), qui décrivent localement la distribution des trois classes de taux de recouvrement i.e. de densité de l'herbier. L'image utilisée pour ce travail est une scène multispectrale Formosat-2 à $8 \mathrm{~m}$ de résolution acquise sur le Bassin d'Arcachon le 18/08/12. Les données de terrain ont été collectées en septembre 2012. Formosat-2 a une revisite temporelle journalière ce qui maximise les chances d'obtenir une image lors d'une grande marée basse sur les quelques créneaux favorables pendant l'été. La revisite temporelle est un critère essentiel pour cette application. Avec une constellation à 2 satellites, les données Pléiades permettent de proposer une revisite temporelle satisfaisante et de gagner en résolution spatiale ce qui est utile pour détecter des patchs d'herbier métriques.

Les données de terrain permettent de calibrer l'arbre de décision. Au préalable, seule la zone d'emprise de l'herbier est conservée en masquant les zones extérieures (chenaux en eau, schorre, couvert d'huitres) y compris celles dont la signature spectrale est proche de l'herbier (végétation du schorre, huitres) pouvant conduire à de la confusion. Cette approche empirique a été mise en œuvre avec succès pour la réalisation de la cartographie de l'état de l'herbier de zostères du Bassin d'Arcachon en 2012 (Lafon, 2012). Elle est également déployée sur les sites de la baie de Marennes Oléron et de l'île de Ré en 2014 (études en cours).

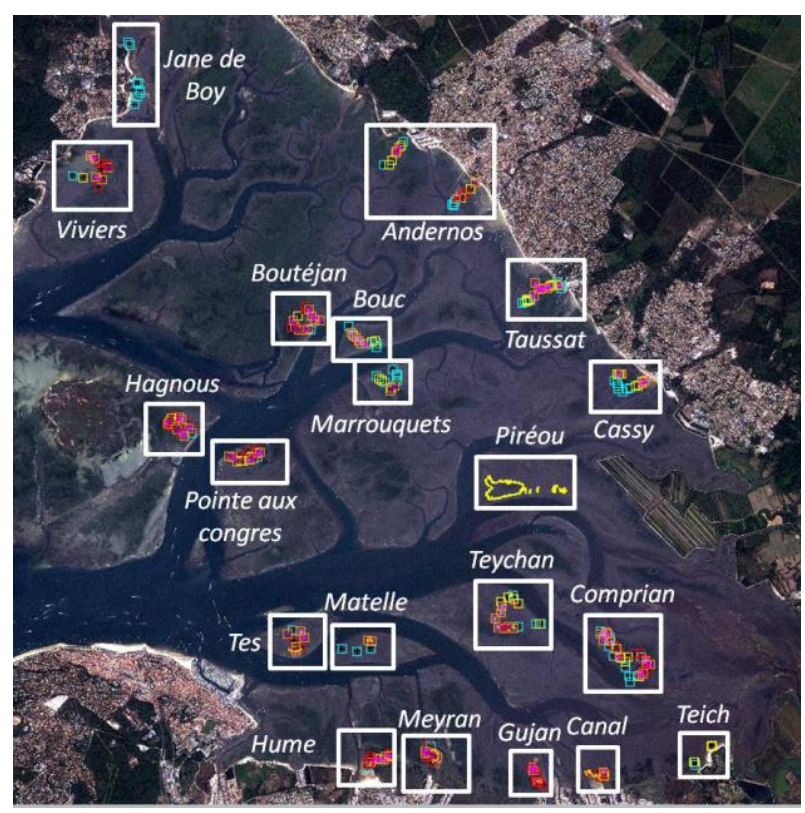

Figure 1 : Localisation des données de terrain servant à l'élaboration de la cartographie de l'herbier à Zostera noltii du bassin d'Arcachon à partir d'une image Formosat-2.

\subsection{Cartographie des lagunes}

La stratégie de cartographie développée dans le cadre du programme SYNIHAL (TOSCA CNES) repose sur la fusion de données HR/THR multi-temporelles optiques et radar. Cette stratégie s'est en effet révélée très performante pour la cartographie de l'occupation du sol en domaine agricole (Chust et al., 2004, McNairn et al., 2010) et les images radars et optiques montrent des propriétés très complémentaires pour la cartographie intertidale (Gade et al., 2011, Kim et al., 2011, Bargain et al., 2013). Elle permet de produire une cartographie exhaustive des faciès biologiques et sédimentaires présents sur les platiers intertidaux (substrats nus, herbiers de plantes aquatiques, macrofaune benthique : i.e. huitres, moules, crépidules, hermelles; végétation du schorre). Cette application adresse les besoins en cartographie de type Natura 2000. Deux approches de fusion ont été testées à partir de données SPOT-5 multispectrales $(10 \mathrm{~m}$ ) et TerraSAR-X (Stripmap 2.75 m) de la base de données Kalideos Littoral. La première approche s'appuie sur une concaténation des bandes optiques de deux images SPOT-5 d'été et d'hiver et d'une image TerraSAR-X (Dehouck et al., 2012). La seconde approche exploite les résultats de deux classifications menées de manière indépendante sur les données optiques et radar (Clos, 2013 ; Lafon et al., 2014a). Plusieurs classifieurs ont été testés sur les images de 2011 de la BD Kalideos: Maximum de Vraisemblance, Distance de Mahalanobis, SVM (Support Vector Machine), réseaux de neurones, et ICM (Iterative Conditional Mode). La meilleure classification est obtenue en combinant les résultats d'une classification par Mahalanobis sur 4 images SPOT-5 du printemps 2011 (3/03, 8/03, 22/03, 1/04) avec une classification par ICM sur 2 images TerraSAR-X $(7 / 02,21 / 11)$.

\subsection{Cartographie des prés-salés}

Les habitats des prés salés sont fréquemment cartographiés sur le terrain, dans le cas où une reconnaissance des espèces est nécessaire. La télédétection aéroportée commence également d'être utilisée (Hladik et al., 2013), quand les approches satellites restaient jusqu'à présent adaptées à une approche assez grande échelle (Lafon et al., 2014a). Le lancement des satellites Pléiades à THR permet d'espérer de nouveaux développements d'applications à la cartographie des prés salés, préservant la reconnaissance des espèces ou groupement d'espèces propice à la connaissance de l'écologie de ces milieux fragiles et préservés. Quatre images Pléiades multispectrales à $2 \mathrm{~m}$ de résolution datant des 14 et 25 Avril, 21 Août et 4 Décembre 2013 ont été exploitées afin d'évaluer la pertinence d'une approche de cartographie des groupements végétaux des prés-salés par satellite pour répondre au besoin des gestionnaires de la Réserve Naturelle Nationale des prés salés d'Arès Lège Cap Ferret. Les données Pléiades utilisées proviennent de la RTU.

Des données de terrain constituées de 452 relevés floristiques (collectés de juin à septembre 2011) ayant permis de réaliser la cartographie des habitats de la réserve (Réveillas et al., 2012) sont exploités comme parcelles d'entrainement de la classification et pour la validation.

En plus des bandes multispectrales, des indices de végétation (NDVI) et de présence d'eau (NDWI) ont été calculés pour chaque date ; créant ainsi un total de 24 bandes spectrales. $\mathrm{La}$ signature radiométrique moyenne de chaque relevé floristique a été extraite dans le but de déterminer les variables séparant le mieux les 8 groupements végétaux. Finalement, 12 bandes constituées par les canaux Proche Infrarouge (B4), NDVI et NDWI ont été sélectionnées. Une image 
composite a été produite, à partir de laquelle a été réalisée une Classification Supervisée Interactive. Les données d'entrainement sont composées de la moitié des relevés floristiques identifiant les 8 groupements végétaux ainsi que les substrats typiques (vasière, sable) et les chenaux en eau. La seconde moitié des relevés floristiques est utilisée pour la validation de la cartographie produite.

\subsection{Suivi de l'évolution des paysages littoraux}

Les données Pléiades sont également exploitées dans le cadre du programme de recherche ayant pour objet le suivi de la dépoldérisation accidentelle survenue à l'lle Nouvelle (estuaire de la Gironde) lors de la tempête Xynthia en février 2010 (Lafon et al., 2014b). Des scènes SPOT-5 d'archive précédant la tempête Xynthia et des images Pléiades post-événement ont été réunies pour retracer le film de l'évolution de l'île suite à la rupture des digues, et suivre la transformation des terres agricoles en vasières et en zones humides littorales, depuis lors soumises à l'action de la marée.

Quatre scènes SPOT-5 des 12/12/09, 25/06/10, $23 / 09 / 11$ et 20/02/12 (acquises dans le cadre du projet INFOLITTORAL-1), et 4 scènes Pléiades des 25/07/12, 19/04/13, 13/08/13, 16/01/14 (acquises via le programme ISIS) constituent la base de données image sur l'lle Nouvelle. Les images Pléiades permettent de suivre l'évolution des objets géomorphologiques d'intérêt (la brèche, la coursive i.e. le nouveau chenal de marée créé à l'intérieur de l'île) ainsi que la dynamique des paysages et du couvert végétal (par le biais du NDVI).

\section{Résultats}

\subsection{Herbier de zostères}

La Figure 2 présente la cartographie de l'herbier à zostères réalisée à partir d'une image Formosat-2 et d'une approche empirique exploitant des observations de terrain. La carte indique la distribution spatiale de l'herbier selon les trois classes de densité voulues dans le cadre de la DCE : herbier à faible recouvrement (0$25 \%$, en vert tendre), herbier à recouvrement moyen (25-75\%, en vert fluo), herbier dense à fort recouvrement (75-100\%, en vert foncé).

La procédure de validation est réalisée en comparant la carte produite avec les observations GPS de terrain (matrice de confusion). La précision globale de la carte est de $70 \%$. La matrice de confusion indique une sousestimation du taux de recouvrement pour les classes 0 $25 \%$ et $25-75 \%$ liée aux effets de mélange intrapixellaire induits par la résolution décamétrique de l'image et également à la présence de flaques d'eau sur le terrain qui affecte la signature radiométrique notamment dans le proche infrarouge. La présence de macro-algues dont la signature radiométrique est très semblable à l'herbier conduit à une sur-estimation du taux de recouvrement. Après nettoyage de la couche d'information sous SIG (fusion des polygones, suppression des polygones isolés de petite taille) et correction à dire d'expert (lorsque des observations de terrain sont disponibles), la précision de la carte atteint $86 \%$.

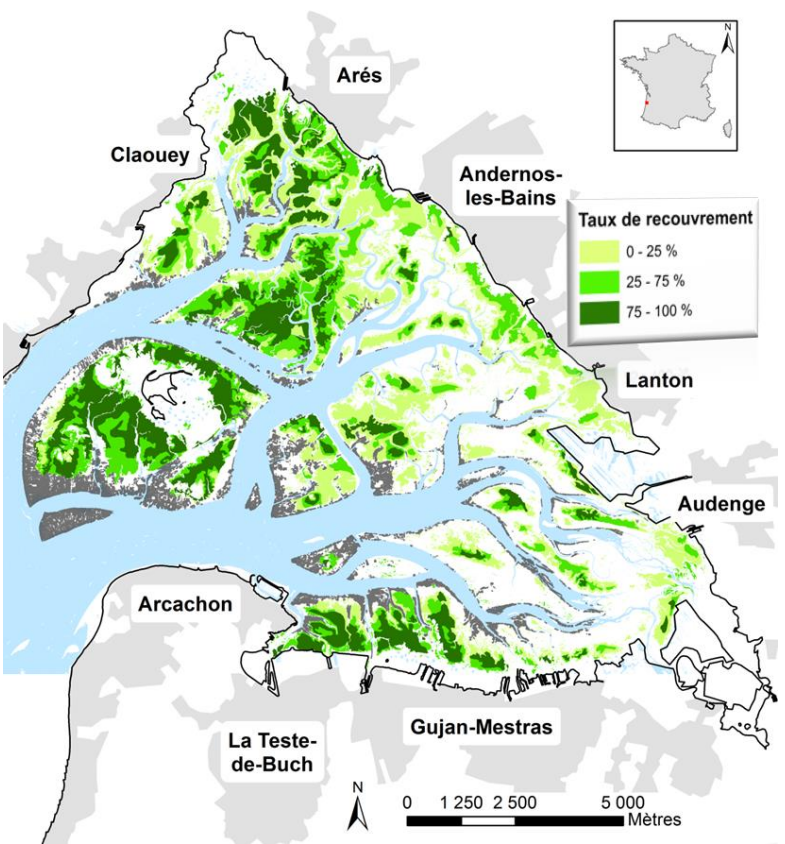

Figure 2: Cartographie de l'herbier à Zostères du Bassin d'Arcachon à l'été 2012 réalisée à partir d'une image Formosat-2

D'un point de vue thématique, la cartographie 2012 de I'herbier indique un déclin de la superficie de l'herbier de l'ordre de $7 \%$ par rapport à celle de 2007 (Lafon, 2012) touchant essentiellement la partie orientale du Bassin d'Arcachon. Entre ces deux dates, la superficie de l'herbier de densité moyenne (25-75\%) recule de $17 \%$, celle de l'herbier dense $(75-100 \%)$ de $25 \%$; a contrario, la superficie de l'herbier peu dense progresse de l'ordre de $28 \%$.

\subsection{Habitats intertidaux}

La Figure 3 présente la cartographie bio-sédimentaire du Bassin d'Arcachon produite par une classification multi-temporelle exploitant des données optiques SPOT-5 et radar TerraSAR-X. Cette stratégie de classification exploite la richesse spectrale des deux sources d'image et la variabilité saisonnière des signatures radiométriques en particulier celles des plantes du schorre et des herbiers de zostères. II en résulte une discrimination réussie entre la végétation intertidale (herbier) et la végétation du schorre (prés salés) ainsi qu'une détection parfaite des couverts d'huitres du fait de leur signature radar bien spécifique (Dehouck et al., 2012).

Cette approche délivre des cartographies dont la précision est de l'ordre de $80 \%$ :

- la restitution des 7 classes majeures décrivant les grands types de substrat et ensembles végétaux de la lagune d'Arcachon par une approche simple combinant 2 images optiques d'hiver et d'été et une bande radar TerraSAR- $\mathrm{X}(\mathrm{HH})$ produit une carte avec une précision de $80 \%$ (Dehouck et al., 2012) :

- la restitution de 17 classes décrivant plus finement les communautés végétales supratidales du schorre (Figure 3) par une fusion des résultats des classifications " optique » et « radar » produit une carte avec une précision de $81 \%$ (Lafon et al., 2014a). 
La seconde approche a exploité l'ensemble de la base 2011 sur le Bassin d'Arcachon (BD Kalideos).

Ce travail révèle que le facteur le plus important est l'obtention de données satellite aux dates clés suivant le cycle phénologique des plantes. Cette approche

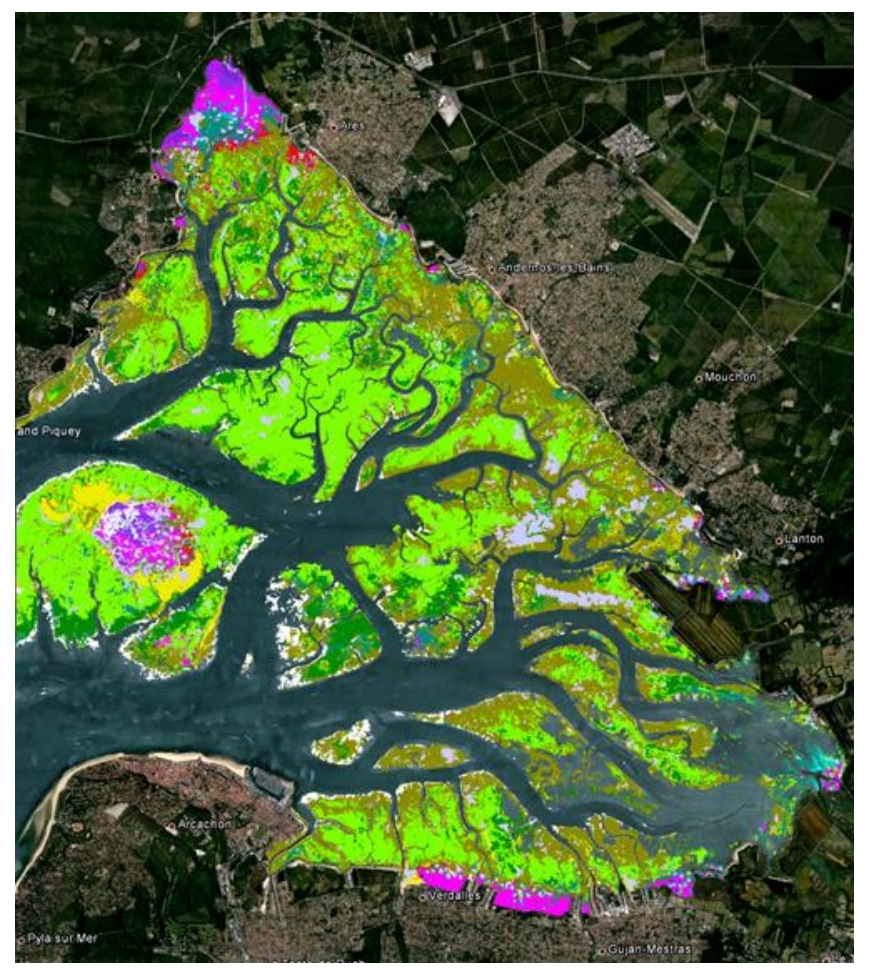

\subsection{Habitats des prés-salés}

Les habitats des prés salés sont classés suivant 6 groupements végétaux (notés de $A$ à $F$ ) qui constituent les prés salés d'Arès Lège Cap Ferret:

- A : haut schorre (association Agropyrion pungentis, code Natura 2000 Habitat 1330-5) caractérisé par le chiendent du littoral et le baccharis ;

- B : haut schorre (association Agropyrion pungentis, code 1330-5) caractérisé par la spartine versicolor et les roseaux Phragmites ;

- C: schorre moyen (association Halimionion portulacoidis, code 1330-2) obiones, limonium commun et joncs maritime ;

- D : bas schorre (association Salicornion dolichostachyae, code 1310-1) caractérisé par les asters, les salicornes vivaces et la soude maritime;

- E : haute slikke (association Spartinion maritimae, code 1320-1) caractérisé par des spartines maritime et le triglochin maritime ;

- F: slikke (association Spartinion anglicae) à spartines anglaises et salicornes.

La précision globale de la cartographie obtenue est de $71 \%$ (coefficient de Kappa de 0,65, Tableau 1). La carte produite (Erreur ! Source du renvoi introuvable.) met bien en évidence la zonation des associations végétales typiques des prés salés. Elle est conforme aux relevés de terrain. Les résultats sont bons à très bons sur la plupart des associations végétales, à l'exception des groupements D $(34 \%$ du point de vue du producteur de la carte) et $\mathrm{E}$ ( $58 \%$ du point de vue de l'utilisateur). de données satellitaires optiques et radar de l'année multi-temporelle multi-source mérite maintenant d'être appliquée à des données THR Pléiades et bientôt Sentinel-1/2.

Figure 3 : Cartographie de l'estran et prés-salés du Bassin d'Arcachon par fusion de données SPOT-5 et TerraSAR-X (Clos, 2013 ; Lafon et al., 2014a).

Tableau 1 : Validation de la classification des prés salés d'Arès

\begin{tabular}{cccccccc}
\hline & A & B & C & D & E & F & $\begin{array}{c}\text { Préc. } \\
\text { Utilisat }\end{array}$ \\
\hline A & 87 & 6 & 0 & 0 & 0 & 0 & $94 \%$ \\
B & 32 & 108 & 17 & 4 & 0 & 0 & $67 \%$ \\
C & 0 & 5 & 94 & 29 & 5 & 2 & $69 \%$ \\
D & 0 & 0 & 9 & 41 & 4 & 2 & $73 \%$ \\
E & 0 & 0 & 0 & 39 & 71 & 12 & $58 \%$ \\
F & 0 & 0 & 0 & 6 & 29 & 95 & $73 \%$ \\
$\begin{array}{c}\text { Préc. } \\
\text { Prod. }\end{array}$ & $73 \%$ & $91 \%$ & $78 \%$ & $34 \%$ & $65 \%$ & $86 \%$ & $71 \%$ \\
\hline
\end{tabular}

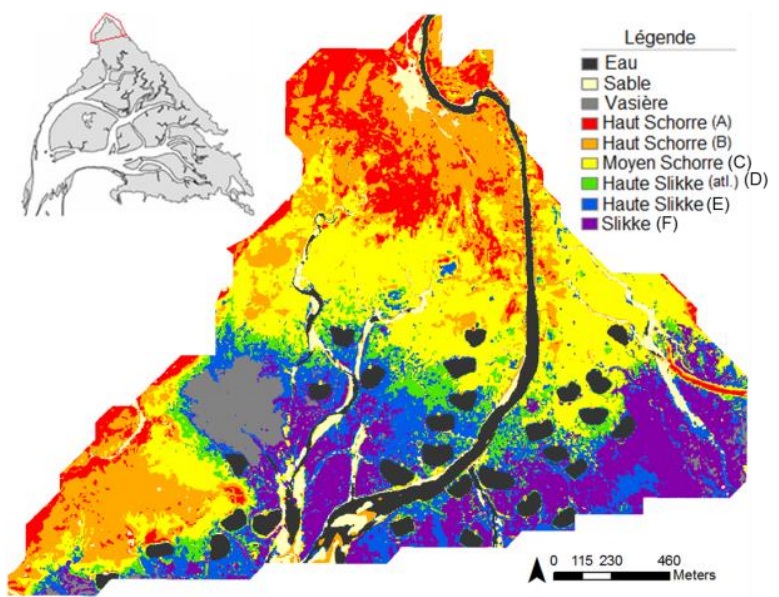

Figure 4 : Cartographie des habitats de la Réserve Naturelle des prés-salés d'Arès Lège Cap Ferret par classification supervisée à partir de 4 images Pléiades de l'année 2013 (Yeo et al., 2014), (C) CNES (2013), distribution Airbus DS / Spot Images..

Ceci peut s'expliquer par le caractère spatialement complexe de l'habitat $D$ qui est un habitat transitoire imbriqué entre le schorre moyen et la haute slikke. L'habitat $E$ se confond lui avec l'habitat $F$ ce qui peut s'expliquer par les fortes similarités floristiques entre ces deux communautés végétales (Spartine maritime vs Spartine anglaise). De ce point de vue, les données radar THR sont utiles pour dissocier les habitats E-F à spartines (disposant d'une signature radar bien spécifique) des autres habitats du schorre. 
En conclusion, du point de vue des botanistes, cette approche associant données THR Pléiades et relevés de terrain est très prometteuse pour le suivi et la cartographie de la végétation des prés salés.

\subsection{Evolution des paysages}

La Figure 5 montre la séquence d'images Pléiades collectées afin de suivre l'évolution de l'île Nouvelle depuis l'ouverture d'une brèche en février 2010 pendant la tempête Xynthia. Avec des images de résolution métrique, on peut observer la dynamique d'objets de petite taille, comme la brèche elle-même, ainsi que des évolutions mineures ou lentes, comme celles affectant depuis peu les berges de la coursive, ce chenal nouvellement créé au sein de l'île. En 3 ans entre 2010 et 2013 , la coursive a progressé très rapidement vers le sud sur une distance de $2.3 \mathrm{~km}$ depuis la brèche. Inexistante à l'origine, cette coursive chenalise les flux de marée entrants et sortants de l'île. Elle est relativement stable depuis 2013 avec une érosion

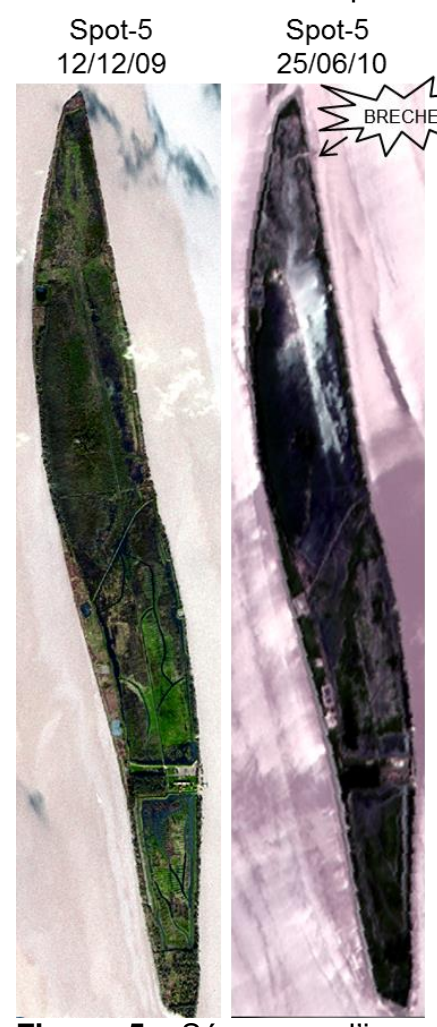

Spot-5 Spot-5
23/09/11 20/02/12
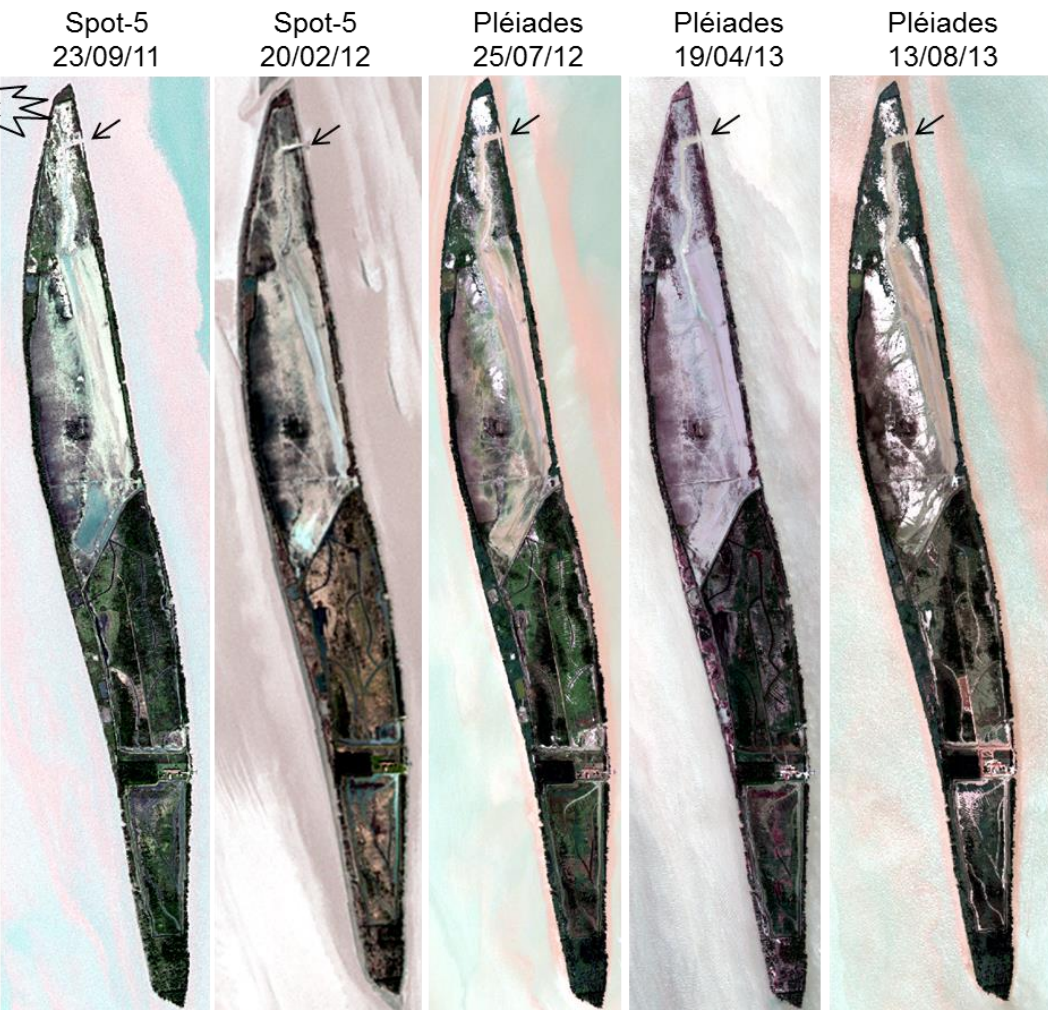

Pléiades $16 / 01 / 14$

Figure 5: Séquence d'images SPOT-5 et Pléiades retraçant la dynamique de changement des paysages depuis l'ouverture d'une brèche au nord de l'lle Nouvelle (estuaire de la Gironde) en février 2010, ( CNES (2012, 2013, 2014), distribution Airbus DS / Spot Images.

\section{Conclusions}

L'imagerie satellite est adéquate pour cartographier les couverts sédimentaires et biologiques des environnements littoraux (herbiers de plantes aquatiques, macrofaune benthique, végétation du schorre). Les stratégies de cartographie développées sont multiples selon les spécifications émises par les gestionnaires du littoral. Elles s'appuient sur des données multi-source (optique/radar) et multitemporelles. Les données radar sont particulièrement sensible de sa rive orientale. Avec l'inondation marine, peuplements d'arbres et les communautés végétales basses ont changé : destruction des frênaies, creation de vasières intertidales par les sédiments déposés à chaque marée, reconquête du milieu par une végétation halophile typique des marais littoraux superficie du couvert végétal originel constitué par les agricoles a reculé de 33 ha entre 2009 et juille 2012 ce qui représente une perte d'environ la moitié de la superficie de la partie nord de l'île. Depuis l'été 2012 2012 et 2013) par une végétation plus riche en termes de biodiversité que la végétation originelle. Lafon et al. (2014b) ont dressé récemment un état des connaissances des processus en jeu et des impacts engendrés par la dépoldérisation sur la dynamique la flore. 
du relief, végétation pionnière ou de transition). La résolution métrique permet de diminuer les effets de mélange intra-pixellaire, responsables de la sousestimation du taux de recouvrement de l'herbier de zostères et d'une discrimination imparfaite de certaines associations végétales, et ainsi d'améliorer la précision des cartographies produites. De plus, la constellation Pléiades offre une capacité de revisite temporelle satisfaisante au regard de la contrainte liée à l'acquisition d'images à marée basse sur des créneaux courts de quelques jours seulement.

\section{Références}

Bargain, L., Robin, M., Méléder, V. Rosa, P., Le Menn, E., Harin, N., Barillé, L., 2013. Seasonal spectral variation of Zostera noltii and its influence on pigmentbased Vegetation Indices. Journal of Experimental Marine Biology and Ecology, 446, 86-94.

Chust G., Ducrot D., Pretus J.L., 2004. Land cover discrimination potential of radar multitemporal series and optical multispectral images in a Mediterranean cultural landscape. International Journal of Remote Sensing, 5 (17), 3513-3528.

Clos G., 2013. Exploitation de l'aspect multi-temporel de la base de données Kalideos Arcachon pour la cartographie multi-thématique en domaine littoral. Rapport de Master 2, Université Bordeaux 3.

Dehouck A., Lafon V., Baghdadi N., Marieu V., 2012. Use of optical and radar data in synergy for mapping intertidal flats and coastal salt-marshes (Arcachon lagoon, France). IGARSS 2012. IEEE International Geoscience and Remote Sensing Symposium, München, Germany, 2853-2856.

Gade M., Stelzer K., Kolhus J., 2011. On the use of multi-frequency SAR imagery for a surveillance of the Wadden Sea ecosystem on the German North Sea coast. In Proceedings of the 4th TerraSAR-X Science Team Meeting, nb pages, available on http://sss.terrasar-x.dlr.de/

Kim D., Choe B., Moon W.M., Park S., Hwang J., Oh Y., 2011. Detection of groundwater discharge and oyster reef in tidal flat using synthetic aperture radar. In Proceedings of the 4th TerraSAR-X Science Team Meeting, available on http://sss.terrasar-x.dlr.de.

Lafon V., 2012. Cartographie de l'herbier à Zostera noltii du bassin d'Arcachon par télédétection spatiale. Rapport ADERA/Agence de l'Eau Adour Garonne.

Lafon V., Dehouck A., Clos G., Ducrot D., Sellé A., 2014a. Multi-temporal and multi-sensor classification applied to intertidal flat mapping. EARSeL $20145^{\text {th }}$ Land Use and Land Cover workshop, Berlin, Allemagne.

Lafon V., Kervella S., Lechene A., Sottolichio A., Rimond F., Fourcade S., Cardonnel S., Alard D., 2014b. Restauration écologique d'un marais intertidal : exemple de la naturation par dépoldérisation de l'île Nouvelle (estuaire de la Gironde, Aquitaine, France). Actes des 13èmes Journées Nationales Génie Côtier Génie Civil, Dunkerque, France, 11 p.
McNairn H., Champagne C., Shang J., Holmstrom D., Reichert G. (2009). Integration of optical and Synthetic Aperture Radar (SAR) imagery for delivering operational annual crop inventories. ISPRS Journal of Photogrammetry and Remote Sensing, 64, 434-449.

Phinn, S., Roelfsema, C., Dekker, A., Brando, V., Anstee, J., 2008. Mapping seagrass species, cover and biomass in shallow waters: An assessment of satellite multi-spectral and airborne hyper-spectral imaging systems in Moreton Bay (Australia). Remote Sensing of Environment, 112, 3413-3425.

Reveillas M., Alfonsi E., Alard D., 2012. Typologie et cartographie des habitats naturels du compartiment sous influence tidale-RNN des Prés salés d'Arès et de Lège Cap-Ferret. Rapport, Université de Bordeaux/ONCFS, $61 \mathrm{p}$.

Yeo S., 2014. Habitat mapping and phytosociological classification of saltmarsh vegetation combining multispectral images with field data. Rapport de Master, Université de Bordeaux, France.

Yeo S., Reveillas M., Alfonsi E., Fievet V., Allou J., Steinmetz J., Paris A., Brun S., Lafon V., Marie-Lise Benot M.-L., Didier Alard D., 2014. Cartographie de la végétation des prés salés d'Arès par l'utilisation conjointe de données de terrain et d'images satellites multi-spectrales. Conférence "Suivis des Prés salés. Quels descripteurs pour quels objectifs de conservation ?", 19-20 juin 2014, Agon-Coutainville, France. 